\title{
Tuberculosis Spine in Neonate: A Case Report
}

\author{
Goh Teik Chiang*1,2, Teo Khai Ling² and Saw Xue $\mathrm{Yi}^{2}$ \\ ${ }^{1}$ Department of Orthopaedics and Traumatology, Hospital Universiti Kebangsaan Malaysia, Malaysia \\ ${ }^{2}$ Department of Orthopaedic and Traumatology, Melaka Hospital, Malaysia
}

Received: 归: October 11, 2018; Published: 眥: October 26, 2018

*Corresponding author: Goh Teik Chiang, Department of Orthopaedics and Traumatology, Hospital Universiti Kebangsaan Malaysia, Kuala Lumpur, Malaysia

\begin{abstract}
Spinal tuberculosis, TB prevalence is increasing across the world but with very little to almost zero data regarding its prevalence in neonates. The complications of spine TB can be as devastating as causing irreversible paraplegia, bone destruction and severe spine deformity which then lead to spinal cord decompression, cardiopulmonary dysfunction. Detecting spine TB in infants at early stage might be difficult as the disease often present with vague symptoms with neurological deficit only manifested at the later stage as demonstrated in our case. Therefore, a clear background history is very crucial in treating any patients with possibility of having TB spine. In many cases, medical treatment alone has good outcome and even improve neurological involvement if treated early. More study however, is required to assess the long-term outcome of Spine TB in neonates and children. We report a case of 2 months old female infant sustained extrapulmonary TB with spinal TB. This case emphasizes on difficulty in diagnosis and challenge in treating neonate TB spine in our centre.
\end{abstract}

Keywords: Spine tuberculosis (TB); Neonates; Extrapulmonary; Antituberculous treatment

\section{Introduction}

Spine TB is a destructive form of tuberculosis [1]. There is a report that suggest the incidence is increasing in developed countries primarily from immigrants from endemic nations. The exact prevalence of spine TB however is not known. About $10 \%$ of patient with extra pulmonary TB have skeletal involvement and spine TB accounts for $50 \%$ out of this number followed by hip and knee [2]. Spinal TB is a very dangerous type of skeletal TB as it can be associated with neurological deficit due to compression of the adjacent neural structures and significant spinal deformity [3]. Therefore, early detection of spine TB is very crucial. Data on childhood musculoskeletal TB however is very rare. The minimum time required for osteoarticular tuberculosis to manifest is postulated as one year after the primary infection hence it is extremely unusual to present before one year of age $[4,5]$.

\section{Case Report}

A two months old female infant presented to us with persistent fever and cough. This patient was initially suspected to have pneumonia with MRSA bacteremia. She was treated with IV Azithromycin and IV Vancomycin for one week. Serial chest X ray was done and showed persistent unresolved right upper lobe consolidation which is highly suspicious of complicated pneumonia or abscesses (Figures $1 \& 2$ ). On examination gibbus deformity was noted at the back of the patient (Figure 1). An urgent MRI was done to distinguish diagnosis between spondylodiscitis or TB spine. Clinical examination and radiology investigation strongly suggest for TB spine as destructive bone changes were noted involving T7, T8 and T9 vertebra body (Figure 3). Further history reveals that the patient was taken care by an Indonesian care taker since birth. However, further investigations and history are needed to confirm the source of TB contact.

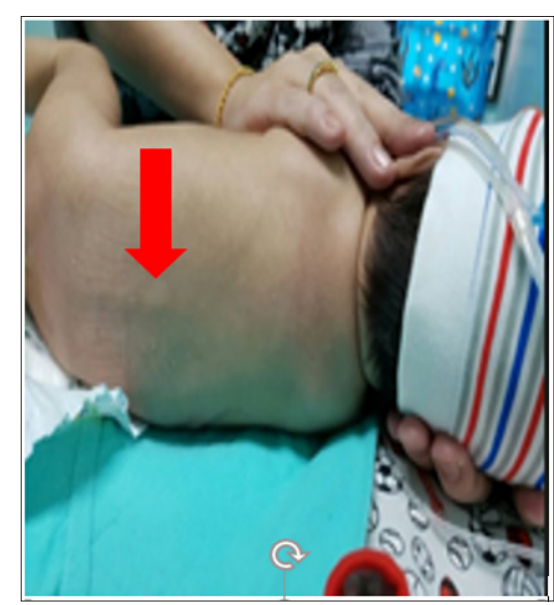

Figure 1: Showed gibbus deformity. 


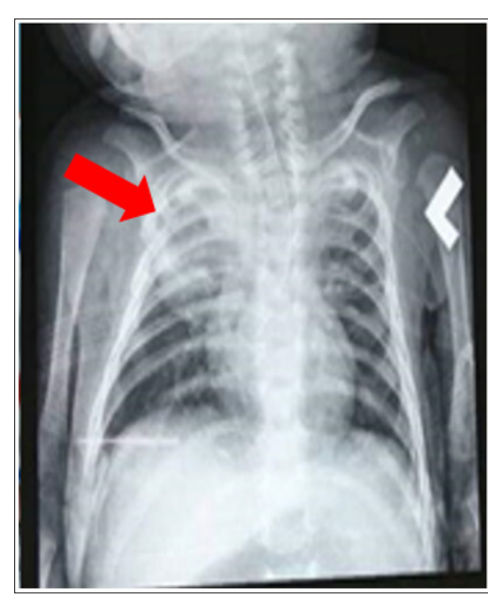

Figure 2: Showed apical opacity of right lung.

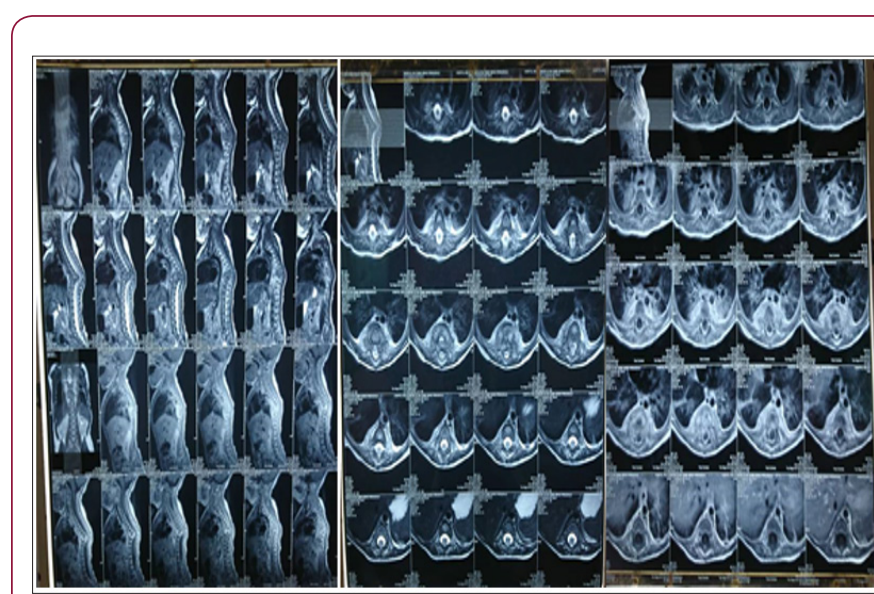

Figure 3: MRI showed destructive body vertebra of T7, T8 and $\mathrm{T} 9$.

\section{Discussions}

Spinal tuberculosis occurs mostly in children and young adults but extremely rare in neonates $[5,6,7]$. The most common differential diagnosis in neonates and children is vertebral osteomyelitis. At an early stage patient might present with variable and unspecific symptoms mainly back pain, fever and weight loss. These symptoms however are hard to detect in infants which might attribute to the delay of the diagnosis [1]. Just like, TB spine, children with vertebral osteomyelitis also often presented with vague symptoms. The overall incidence of vertebral osteomyelitis is 1 in every 250000 . The estimated prevalence among the children is however not available due to limited data and literature $[3,4]$. At the later stage of TB spine patients might also presented with sensory disturbance, bowel and bladder dysfunction. In adult, the spread of the disease is usually secondary to the spread of infection through vertebral body. In children, the infection route is through hematogenous spread of $\mathrm{M}$. tuberculosis from a primary site of infection which is often unknown into the dense vasculature of cancellous bone of the vertebral bodies [2].

A research has shown that in $50 \%$ of cases concomitant active pulmonary disease is present. The destruction of the intervertebral disc space and the adjacent vertebral bodies collapse of the spinal elements, and anterior wedging lead to the characteristic angulation and gibbus as seen in the infant in our case. In this case, Gibbus is the palpable deformity formed due to the involvement of multiple vertebrae which results in acute kyphosis [2,4]. In TB spine, the upper and lower thoracic spine are the most commonly involved sites whereas in vertebrae osteomyelitis, lumbar sacral is found as the most affected site which accounts $75 \%$ out of all pyogenic spondylitis. In most cases of $\mathrm{Tb}$ spine, more than one vertebra is usually affected [3]. One of our challenge in this case was to differentiate spinal TB from pyogenic and fungal vertebral osteomyelitis as well as primary and metastatic spinal tumours because only clinical and radiographic findings are available. Other test like tuberculin skin test, acid fast bacilli along with clear background history play an important role in diagnosing spine TB however it was unable to perform in this case.

$\mathrm{X}$-ray images might be normal in an early disease [1,2]. More than $50 \%$ of bone loss is required before it becomes evident on $\mathrm{X}$-ray. At later stage of the disease, collapse of the intervertebral disc space can be seen when disc involvement happens. At this stage, the disease is often at a very advanced stage with patients having neurological presentations. Common characteristic radiographic findings on $\mathrm{x}$-rays include rarefaction of the vertebral end plates, loss of disk height, osseous destruction, new-bone formation and soft-tissue abscess [2,3]. Often, multiple vertebrae are involved and late fusion or collapse of vertebrae is not uncommon. CT scan might help us to detect TB spine earlier than X-ray. The pattern of bone destruction may be fragmentary in $47 \%$ of the cases; osteolytic in $34 \%$, localized and sclerotic in $10 \%$, and sub periosteal in $30 \%$ cases [2]. CT scan is good in demonstrating any calcification within the cold abscess or visualizing epidural lesions containing bone fragments as well as delineation of encroachment of the spinal canal by posterior extension of inflammatory tissue, bone or disk material, and a great value in the CT-guided biopsy.

In this case, MRI was performed because it is the gold standard for the diagnosis of spine TB in this age group [8]. It is more sensitive than $\mathrm{x}$-ray and more specific than CT in the diagnosis of this disease. MRI allows for the rapid determination of the mechanism for neurologic involvement. The insidious onset of the disease, the smooth margins of the para spinal mass and the rim enhancement of this mass in MRI are the main criteria for distinguishing vertebral tuberculosis from pyogenic spondylitis [2]. As for treatment for spine TB, our main goal is to eradicate the infection, to ensure a good recovery from any neurological deficits and to cure the disease with minimum residual spinal deformity. Anti-tuberculous should be started as early as possible [8]. Majority of patients (about 85-95\%) of spinal tuberculosis respond very well to medical treatment. The treatment response is apparent in form of pain relief, decrease in neurological deficit, and even correction of spinal deformity.

Perinatal TB is usually fatal if untreated. This is our greatest challenge in this case, the infant was empirically commenced on treatment as per national guidelines. Since this is not a common 
disease, no therapeutic trials have determined the optimal treatment regimen and length. Complete recovery has been described following a standard treatment course of 2 months of 4 drugs (isoniazid, rifampicin, pyrazinamide and streptomycin), followed by 4 months of 2 drugs (isoniazid and rifampicin) according to World Health Organization (WHO) [8]. However other regimens for up to 18 months have been described and clinicians should seek expert advice. Treatment length should be determined by clinical condition and response to treatment. We started antituberculous regime according to WHO guidelines in this case. Surgical drainage is indicated when large abscesses are present, especially in the psoas muscle or patient developing neurological deficit on follow-up must undergo surgery to prevent irreversible paraplegia or worsening deformity [5]. Almost 3\% of children with spine TB develops severe kyphosis of more than $60^{\circ}$ [5]. Factors increasing the risk of severe kyphotic deformity are children being below 10 years of age, involvement of three or more vertebral bodies and localization of the lesion in the thoracic spine $[3,7,8]$ as presented in our case. Therefore, long term follows up for this patient is mandatory.

\section{Conclusion}

In summary, the therapeutic goal in children is to heal the tuberculosis with minimum residual deformity and without neurologic sequelae. Therefore, the treatment should be aimed not only at the healing, but also at the maintenance of stability, normal spinal growth, and sagittal alignment of spine by preventing the additional progressive bone destruction and or by hastening the neurological recovery during the treatment period and afterwards. Hence, the children should remain under surveillance follow up until growth phase is complete in this case.

ISSN: 2574-1241

DOI: $10.26717 / B J S T R .2018 .10 .001952$

Goh Teik Chiang. Biomed J Sci \& Tech Res

(c) (P) This work is licensed under Creative Commons Attribution 4.0 License

Submission Link: https://biomedres.us/submit-manuscript.php

\section{Consent Disclaimer}

As per international standard or university standard, patient's written consent has been collected and preserved by the author(s).

\section{Ethical Disclaimer}

As per international standard or university standard ethical permission has been collected and preserved by the authors.

\section{References}

1. Starke JR (2009) Text book of Paediatric Infectious Diseases ( $6^{\text {th }}$ edn.). Vol. 1. Elsevier: Saunders; 2009. Tuberculosis. In: Feigin RD, Cherry JD, Demmler GJ, et al. (Eds.). pp. 1442-1223.

2. Andronikou S, Jadwat S, Douis H (2002) Patterns of disease on MRI in 53 children with tuberculous spondylitis and the role of gadolinium. Pediatr Radiol 32(11): 798-805.

3. Singh SN, Chatt TC, Kumar S, Chauhan V, Pandey A (2016) A Case of Cervical Spine Tuberculosis in an Infant. Journal of Clinical and Diagnostic Research 10(1): TD03-TD05.

4. Mohammed Benzagmout, Boujraf S, Chakour K, Mohammed El Faïz Chaoui (2011) Pott's Disease in Children. Surgical Neurology Interational 2: 1 .

5. Moon MS, Kim SS, Lee BJ, Moon JL (2012) Spinal tuberculosis in children: Retrospective analysis of 124 patients. Indian Journal of Orthopaedics 46(2): 150-158.

6. Garg RK, Somvanshi DS (2011) Spinal tuberculosis: A review. The Journal of Spinal Cord Medicine 34(5): 440-454.

7. Moraes Barros Fucs PM, Meves R, Yamada H (2012) Spinal infections in children: A review. International Orthopaedics 36(2): 387-395.

8. Rasouli MR, Mirkoohi M, Vancaro AR, Yarandi KK, Movaghar VR (2012) Spinal Tuberculosis: Diagnosis and Management. Asian Spine Journal 6(4): 294-308.

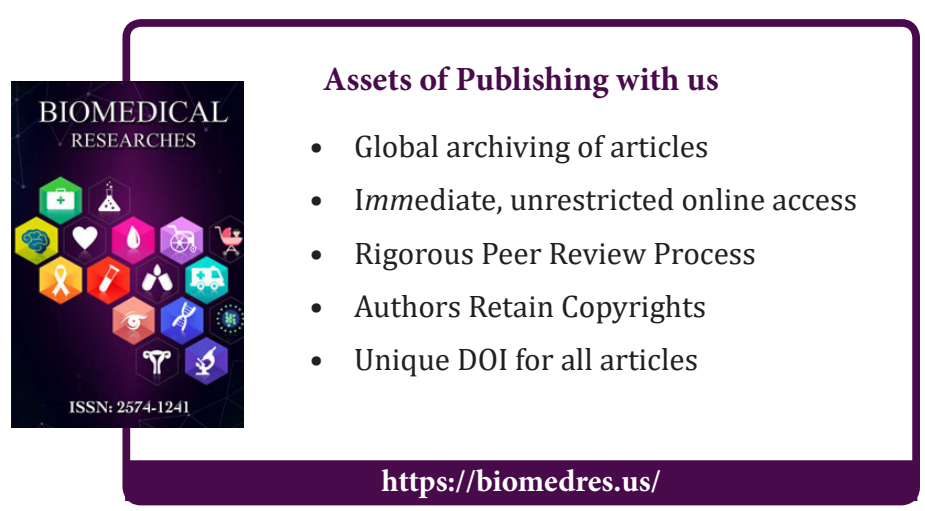

Cite this article: Goh Teik Chiang, Teo Khai Ling, Saw Xue Yi. Tuberculosis Spine in Neonate: A Case Report. Biomed J Sci\&Tech Res 10(3)- 